\title{
Liter per Hour per Kilogram per Meter Squared
}

National Cancer Institute

\section{Source}

National Cancer Institute. Liter per Hour per Kilogram per Meter Squared. NCI

Thesaurus. Code C120739.

A unit of concentration equal to liter per hour divided by kilogram per meter squared. 